\title{
Latent autoimmune diabetes in adults: complication, management and treatment modalities
}

\begin{abstract}
Latent autoimmune diabetes in adults is phenomenal and most commonly referred as "late onset diabetes", initially defined as "non-insulin" dependent diabetes as reported in individuals older than 30 years. Autoimmune diabetes could be a heterogeneous illness which might arise at any age and affects $10 \%$ of people older than 35 years and $25 \%$ below that age. When we compare childhood onset type 1 diabetes with adult-onset autoimmune diabetes, it actually has less Human Leucocyte Antigen (HLA) which is associated with genetic risk and fewer diabetes-associated autoantibodies as well. Despite the presence of islet antibodies at the time of diagnosis, the death progression of autoimmune beta-cell is slow. Subjects with adult onset insulin dependent diabetes mellitus who do not necessitate hypoglycemic agent medical aid for a minimum of six months after diagnosis are demarcated as having LADA. LADA is characterized by serological evidence of islet autoimmunity in the setting of diminished and declining insulin secretion. How and by whom the diagnosis is made and above all on the requirement for exogenous insulin at the time of diagnosis create a distinction between LADA and adult onset type 1 diabetes. In concurrence with demonstrated impaired beta-cell work at diagnosis of diabetes, insulin is the treatment of choice. Although it has closer pathophysiological resemblance to type 1 diabetes, phenotypically LADA is often misdiagnosed and treated as type 2 diabetes due to both lack of knowledge and awareness among physicians to find some standardized diagnostic criteria and started on oral hypoglycemic. This condition of autoimmune diabetes share clinical and metabolic features with both type 1 and type 2 diabetes.
\end{abstract}

Keywords: pancreatic beta-cells, auto antibodies, diabetes mellitus type 1, type 2, anti-GAD, C peptide, HLA, HbA, autoreactive T-cells, insulin, LADA
Volume 7 Issue 3 - 2019

Pallabi Banerjee, Nida Zaman Khan, Sakshi, Tripti Singh, Nagendra Singh and Imteyaz Qamar

School of Biotechnology, Gautam Buddha University, India

Correspondence: Imteyaz Qamar, School of Biotechnology, Gautam Buddha University, Gautam Budh Nagar, UP-201312, India.Email imteyazqamar@gmail.com

Received: April 24, 2019 | Published: May 22, 2019

\section{Introduction}

Diabetes mellitus refers to a group of serious, lifelong condition or disease that affect how your body utilizes sugar (glucose). It is a condition in which the body does not turn out enough insulin or isn't ready to use the insulin effectively. As an outcome, the glucose in the blood (often called blood sugar) turns out to be too high. Maintaining consistent blood sugar level is a critical part of overall health and wellness. The development of diabetes is due to numerous pathogenic processes. These ranges from autoimmune destruction of the pancreatic $\beta$-cells with ensuing insulin inadequacy to irregularities that outcome in resistance to insulin activity. ${ }^{1}$ Diabetes is mainly categorized into two types: TYPE 1 and TYPE 2 . There is another type of diabetes which is most commonly known as Latent Autoimmune Diabetes in Adults or LADA, but is rarest form of diabetes as shown in Figure 1.

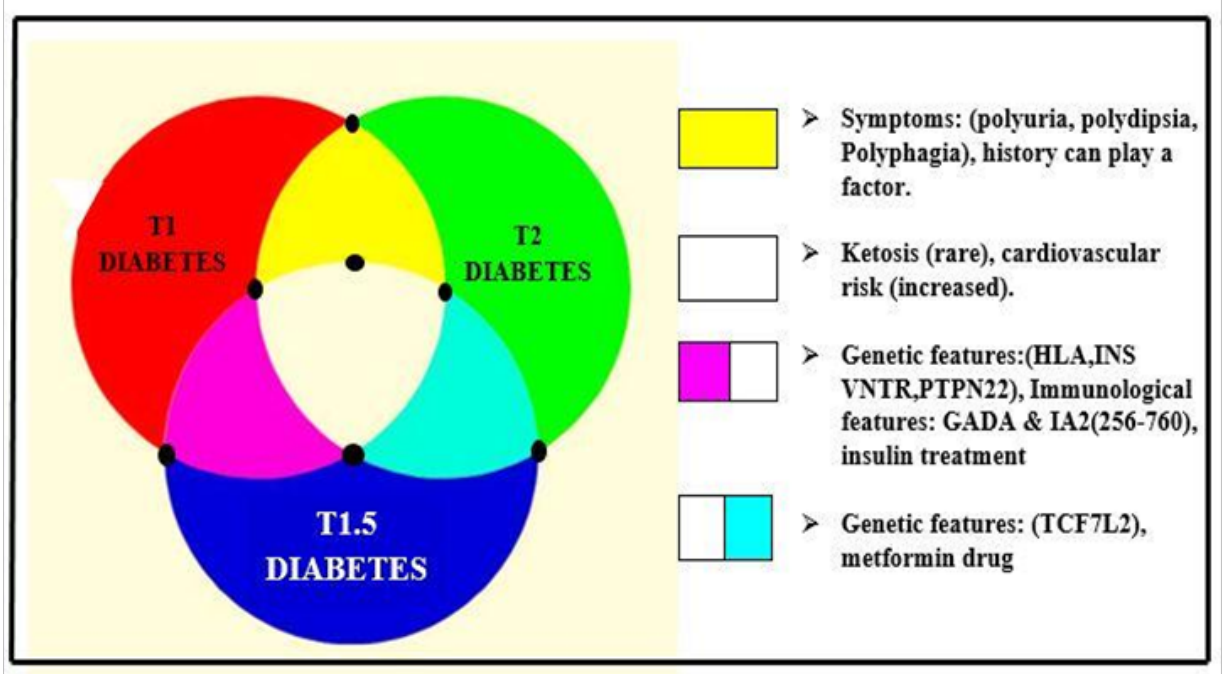

Figure I Common features between TID,T2D, and TI.5. 


\section{Type I diabetes}

Type 1 diabetes is a chronic, autoimmune disorder which is characterized by the body's inability to secrete adequate amount of insulin due to self-destruction of the insulin producing beta cells in the islets of Langerhans within the pancreas. ${ }^{2}$ Patients with type 1 diabetes require lifelong insulin treatment. The contributing factors to the onset of type 1 diabetes are- genetic, environmental and stochastic events. However, its incidence has increased vividly over the last two decades, especially in children less than 5 years old. Once the beta cells are destructed, the patients with type 1 diabetes lose their blood glucose control, which can result in both acute condition (for exampleketoacidosis and severe hypoglycemia) and secondary complications (including heart disorders, blindness and kidney failure) - even with current insulin replacement therapies. ${ }^{3}$

\section{Type 2 diabetes}

Type 2 diabetes is a progressive, metabolic disorder caused by the body's inability to produce or respond to insulin, which is referred to as insulin resistance. It for the most part triggers individuals who are beyond 40 years of age. However type 2 diabetes most often progresses in adults, but kids also can develop type 2 diabetes. ${ }^{4}$ Even if we can determine type 2 diabetes by lifestyle and heredity of genes, insulin sensitivity gets improved only by dietary composition and it helps reduce risks of diabetes and complications as well. ${ }^{5}$

\section{Type I.5 diabetes}

Universally known as latent autoimmune diabetes in adults is a slow progressing form of autoimmune diabetes. ${ }^{6}$ Alike the type 1 diabetes, LADA occurs because the pancreas stops producing adequate insulin. Some patients, specifically kids and youth, may be detected with ketoacidosis at the primary manifestation of the sickness. LADA occurs in $10 \%$ of individuals who are older than 35 years and in $25 \%$ of those who are below among all patients with phenotypic type 2 diabetes. These patients share numerous hereditary and immunological resemblances with type 1 diabetes, proposing that LADA, similar to type 1 diabetes, is an autoimmune disease. It has been demonstrated by Imminent investigators that LADA patients with various islet antibodies develop $\beta$-cell failure within 5 years, while those with just islet cell antibodies (ICAs) or just GAD antibodies (GADAs) generally develop $\beta$-cell failure after 5 years. ${ }^{7}$ Nonetheless in a few adults the residual beta-cell characteristic may additionally hold that is sufficient to save you from ketoacidosis for many years. Ketoacidosis is an extended-time period difficulty of LADA, mainly as soon as dependence on insulin develops. ${ }^{8}$ Sufferers tormented by LADA commonly have worse hemoglobin A1c (HbAlc) degrees than type 2 diabetes patients.

\section{Signs and symptoms}

As reported in diabetes.co.uk, early LADA symptoms may be vague. ${ }^{9}$ they include: feeling tired all the time or feeling tired after meals, foggy headedness, feeling hungry again soon after meals, mood adjustments and irritability. On progression of LADA, a person's ability to produce insulin gradually decreases, and this may lead to more typical diabetes symptoms which typically resembles to symptoms of type 2 diabetes such as ${ }^{10}$ blurred imaginative and prescient, polydipsia (excessive thirst and drinking), polyuria (excessive urination), tingling nerves (especially of hands and feet), weight loss no matter a boom in urge for food.

\section{Genetic features}

The association of LADA to different degrees of autoimmune activities and genetic heterogeneity might be partly different from both type 1 and type 2 diabetes. Unlike classical type 1 diabetes, LADA is also associated with several genetic markers, such as- PTPN22 (1p13.2), STAT4 (2q32.2), CTLA4 (2q33.2), IL2RA (10p15.1), HLA (6p21), INS (11p15.5), SH2B3 (12q24.12), ERBB3 (12q13.2), and CLEC16A (16p13.13). ${ }^{11}$

\section{PTPN22}

Protein tyrosine phosphatase non-receptor type 22 (PTPN22), is a $105 \mathrm{kDa}$ protein that encodes the lymphoid specific tyrosine phosphatase protein Lyp and a powerful negative regulator of T-cell activation and T-cell proliferation, located on chromosome $1 \mathrm{p} 13$ has been associated with LADA. ${ }^{12}$ The mutant allele carried by the associated populations is highly reproducible as indicated by the genetic studies. The functional consequences of a single base change at the DNA level of PTPN22 remain unresolved in spite of these validation studies and genetic association studies with disease in man. ${ }^{13}$

\section{STAT4}

The transcription factor of STAT protein family triggered by the Janus kinase (JAK) family, more or less acts same as signal transducer and activator of transcription 4 (STAT4), located on: 2q32.2-q32.3, which is the long (q) arm of chromosome 2 between positions 32.2 and 32.3. According to several Studies, a normal variation in the STAT4 gene with an increased risk of several autoimmune disorders STAT4 binds to hundreds of sites in the genome, ${ }^{14}$ amongst others to the promoters of genes for cytokines (IFN- $\gamma, \mathrm{TNF}$ ), signaling factors (MYD88) and receptors (IL18R1, IL12rß2, IL18RAP. The $\mathrm{T}$ allele expression is the genetic variant which confers the highest risk of developing autoimmune disorders in the single nucleotide polymorphism (SNP) rs7574865 instead of the G allele and the functional significance of this change remains unclear, as it occurs in the third intron of the STAT4 gene, which is a non-coding region. ${ }^{15}$

\section{CTLA4}

A protein receptor, Cytotoxic T-lymphocyte-associated protein works as an immune checkpoint, (or checkpoint inhibitor) and downregulates immune responses. It is also known as CD152 (cluster of differentiation 152), which is a member of the Ig gene superfamily present on T cells. Several autoimmune disorders including insulin dependent diabetes mellitus are caused due to mutations in this gene. CTLA4 haploinsufficiency is inherited in an autosomal dominant manner, which means that a person only needs an abnormal gene from one parent to have the disease. One normal copy of the gene is not enough to compensate for the abnormal copy as shown in Figure 2. ${ }^{16}$

\section{HLA}

The human major histocompatibility complex gene group, located on the short arm of chromosome 6 (6p21) delivers instructions in the making of a group of related proteins known as the human leukocyte antigen (HLA) complex. HLA types are inherited, and some of them are associated with autoimmune disorders like LADA and other diseases. However, the prevalence of HLA risk halotypes DR4-DQ $\beta 1 * 0302$ and the DR3/DR4-DQ $\beta 1 * 0302$ genotypes were significantly increased in LADA patients and they were much less common than in childhood-onset Type 1 diabetes. ${ }^{17}$ 


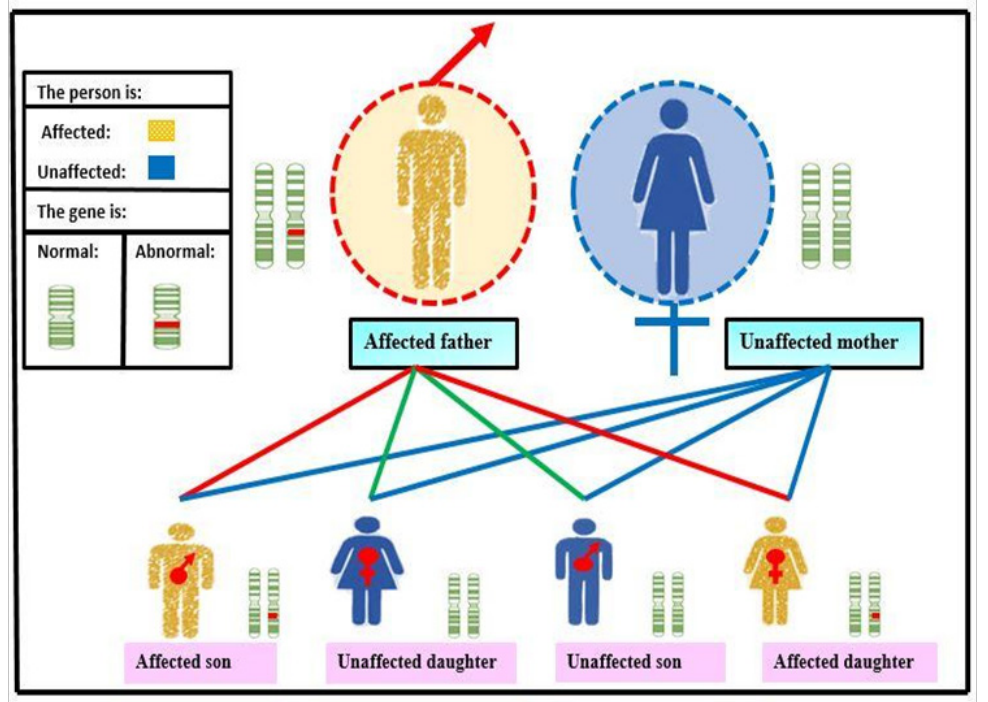

Figure 2 In this example, a man with an autosomal dominant disorder has two affected children and two unaffected children. Women can also pass on the mutation. Credit: U.S. National Library of Medicine.

\section{IL2RA}

Interleukin-2 receptor alpha chain (also called CD25) is a protein, located on short (p) arm of chromosome 10 at position 15.1(10p15.1), which is encoded by the IL2RA gene in humans and associated with LADA. ${ }^{18}$

\section{INS VNTR}

The INS gene gives commands for producing the hormone insulin that is necessary for the maintenance of glucose level within the blood. The precursor of insulin is encoded by the INS gene. ${ }^{19}$ The presence of variable number of tandem repeat (VNTRs) region within the insulin gene (INS) presumably effects the progression of LADA . A study was administered among the Caucasian population and it had been suggested that various INS-VNTR alleles were found in LADA subjects. ${ }^{20}$

\section{Immunological features}

A common feature of autoimmune diseases as seen in type $1 \mathrm{DM}$ is antigen spreading, where resilience to increasingly more islet antigens is lost with the outcome that numerous auto antibodies are available in blood. These antibodies are likewise found in the LADA patients where hostile to GAD and ICA are progressively pervasive and IAA, IA-2A and zinc transporter 8 (ZnT8) are less frequently revealed. ${ }^{21,22}$ The recognition of islet cytoplasmic antigens by Islet cell antibodies (ICA), were first portrayed in recently analyzed T1D patients. ${ }^{23}$ and contain various autoantigens including IA2A and GADA. The neurotransmitter gamma-aminobutyric acid (GABA) which is a neuronal enzyme is synthesized by Glutamic acid decarboxylase (GAD) and is viewed as one of the most grounded autoantigens engaged with activating beta-cell-explicit autoimmunity. The frequency of IgG4 of glutamic acid decarboxylase antibody is increasingly more in LADA patients than type $1 \mathrm{DM} .{ }^{24}$ Autoantibodies particularly IA-2A and ZnT8A tend to disappear, after diagnosis of LADA. ${ }^{6,25} \mathrm{ZnT} 8$ is a multispanning transmembrane protein involved in the accumulation of zinc in the intracellular vesicles. Zinc itself plays an important role in the pancreatic beta cells where fusing of two zinc particles with six insulin molecules leads to the formation of hexamers. In contrast to GAD and IA-2, ZnT8 is extremely $\beta$-cell specific; therefore, the presence of ZnT8As in patients from the Non-Insulin Requiring Autoimmune Diabetes (NIRAD) study demonstrates that the pancreatic beta-cells are targets of the autoimmune process in adult. ${ }^{26}$ Several investigations portrayed contrasts in LADA with respect to irregular DNA methylation in CD4 + T -cells in LADA, ${ }^{27}$ modified Tregulatory cells, ${ }^{28} \mathrm{NK}$ cells, monocytes ${ }^{29}$ and some antigen-responsive T cells. ${ }^{30}$

\section{Clinical features and diagnostic criteria in lada-subjects}

Since LADA is generally mistaken with type 2 diabetes at first, to arrive at diabetes diagnosis, patients usually undergo traditional glucose testing, which is made by a positive test and may be confirmed by a second positive test on a different day. The results are positive if A1c greater than or equal to $6.5 \%$, fasting plasma glucose of greater than or equal to $126 \mathrm{mg} / \mathrm{dl}$ with the symptoms of diabetes or Oral glucose tolerance test (OGTT) has a test value of greater than or equal to $200 \mathrm{mg} / \mathrm{dL}$. At the point when these LADA patients are commenced on injectable insulin, they progress admirably. The best way to affirm LADA is through antibody testing. Carrying out a GADA antibody test is the most widely recognized strategy for diagnosing LADA, yet not all patients have these antibodies. In the extremely beginning periods of LADA, it is conceivable that there are no discernible antibodies, however they can develop after some time. Therefore GADA test alone cannot rule out LADA. ${ }^{31}$ The frequency of LADA is much higher ( 25\%) among patients with type2 diabetes younger than 35 years of age at the time of diagnosis. ${ }^{32,33}$ Despite the fact that amid the first time after detection of diabetes, LADA patients are not insulin requiring within 6 years. In most of such patients, insulin dependency is prompted when $\beta$-cell function is seriously weakened. Furthermore, in contrast to type 2 diabetes, fasting C-peptide is lower in LADA. ${ }^{34}$ Additionally, the level of insulin secretion in LADA is believed to be intermediate between T1D and T2D. Significantly, within a couple of years after LADA is diagnosed, a quick decline occurs in both insulin secretion and stimulated C-peptide secretion..$^{35}$ The clinical parameters that perseveres is that LADA patients are usually lean at diagnosis, ${ }^{36,37}$ alike children with type 1 diabetes. However, this 
view is not supported by the documentation of the BMI in LADA populations . Most investigation of LADA accomplices report a mean
BMI in the overweight or obese categories (BMI $>25.0 \mathrm{~kg} / \mathrm{m} 2)$ Figure $3 .^{38,39}$

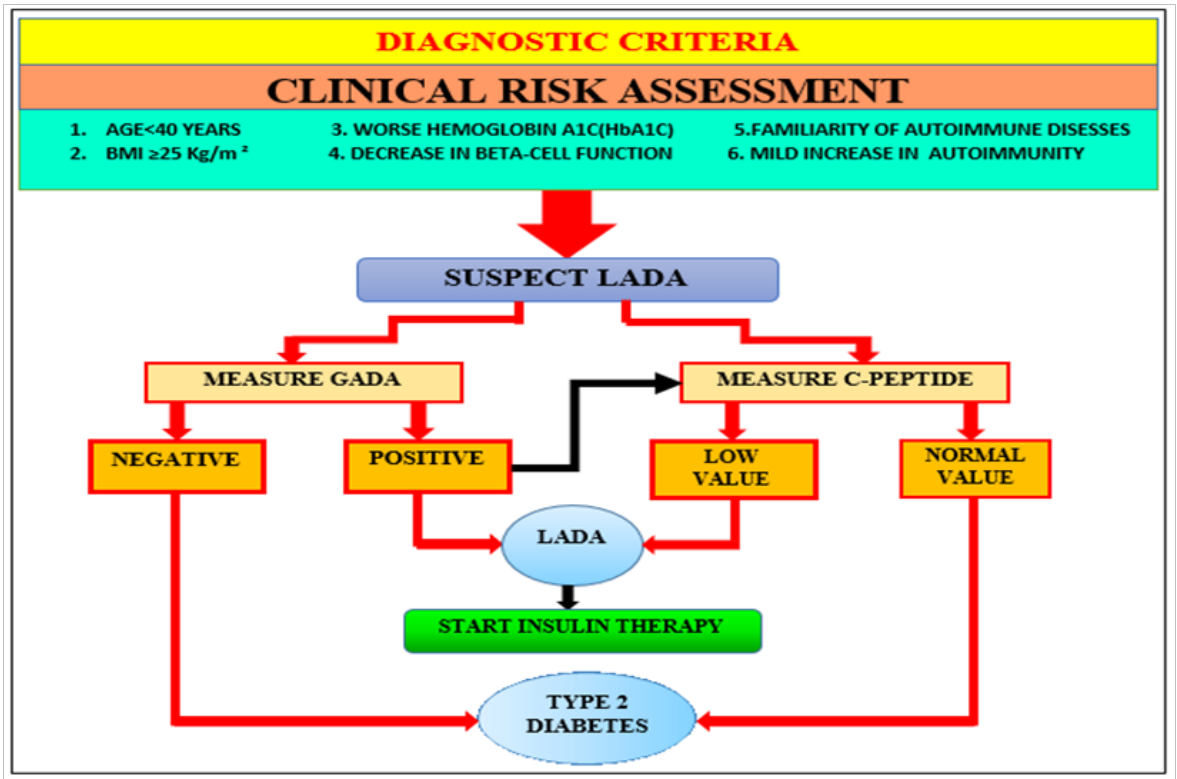

Figure 3 Diagnostic criteria for the assessment of latent autoimmune diabetes in adults (LADA).

\section{Treatment of lada}

Till date no specific guideline for treatment of LADA has been accounted for due to the vague pathophysiology and clinical highlights of LADA. In general, treatment should aim to offer metabolic control and prevent persistent complications. Ongoing administration choices for LADA comprise of restorative way of life changes, oral medications and insulin. ${ }^{40}$ The critical task for the doctor is to abstain from missing a LADA analysis. Once LADA is analyzed, the doctor ought to perceive that some LADA patients require insulin, but due to the similitudes to type 2 diabetes, they may benefit by simultaneous treatment with different oral medicines. Although some healthy b-cell function remains at diagnosis, many LADA patients are initiated on oral medication(s). In any case, usually utilized hypoglycemic medications, for example, sulfonylureas (SUs), metformin, or thiazolidinedione (TZDs) and DPP-4 inhibitors, are beneficial in maintenance of blood glucose level, yet they are utilized only for short-term treatment of LADA. In the long run, insulin treatment is required within 5 years of diagnosis. In this regard, several data showed that insulin treatment, as well as three DPP-4 inhibitors (sitagliptin, linagliptin and saxagliptin) can preserve residual betacell function, whereas sulfonylureas may hasten insulin dependency and ought not be used as a first line treatment for LADA patients[6]. DPP-4 inhibitors are inhibitors of dipeptidyl peptidase that work by lowering blood glucose level without inflicting hypoglycemia (low blood sugar). These medications can likewise enable the pancreas to make more insulin. Sulfonylureas are a category of medications that have been in use for a long time and are commonly used as a second agent to help reduce mealtime blood sugar, for example- Metformin is likely valuable in obese LADA sufferers. ${ }^{41}$ Despite the fact that, insulin treatment is the remedy of desire.

\section{Insulin therapy}

The insulin treatment is important in all patients with entire loss of pancreatic beta cells .GADA positivity is strongly associated with subsequent insulin requirement. ${ }^{42}$ In the UKPDS, $84 \%$ of GADApositive patient's versus $14 \%$ of GADA-negative patients obtained insulin treatment within six years after diagnosis. ${ }^{25}$ However, subjects with latest prognosis of LADA are characterized through some degree of preservation of beta cell characteristics as shown by C-peptide levels, and improvement slower to absolute insulin dependency. Nonetheless, steady information from randomized clinical trials highlight the significance of an early initiation of insulin therapy in LADA irrespective of presence of a few endogenous insulin secretions. The reason behind this technique is to improve metabolic control whilst protecting $\beta$-cells characteristic. ${ }^{43}$

\section{Chronic complications of lada}

Until now, only insufficient records related to the improvement of latent autoimmune diabetic complications have been accounted for in LADA sufferers. The recurrence of ketoacidosis has not been depicted in LADA, however is presumed to be terribly low. Chronic vascular intricacies related with type 1 and type 2 diabetes have been also identified in LADA. ${ }^{44}$

\section{Microvascular and macrovascular complications}

Only few research associated with the event of microvascular complications (nephropathy, retinopathy, and neuropathy) in LADA have been stated similar to type 2 diabetes mellitus. In spite of the fact that a lower risk of microvascular entanglements, especially in a diabetic investigation of Fremantle populace ${ }^{45}$ which stated low 
recurrence (27\%) of microalbuminuria (nephropathy) in LADApatients contrasted with type 2 diabetic patients. Microvascular intricacies in all types of diabetes are believed to be related with hyperglycemia. Glycemic control was comparative in LADA and type 2 diabetes, maybe representing the comparative frequencies of microvascular complications. It has been determined in Chinese population that the predominance of diabetic nephropathy and diabetic retinopathy were altogether lower in patients diagnosed less than 5 years with LADA than in patients with type 2 diabetes. ${ }^{46} \mathrm{~A}$ lower risk of macrovascular intricacies - including coronary illness, stroke, and peripheral artery disease could be proposed based on the more advantageous metabolic profile of patients with LADA in respect to those with T2DM. The autonomous relationship of hypertension, hyperlipidemia, obesity and hyperglycemia with macrovascular sickness were less common in LADA than in patients with type 2 diabetes. $^{47}$

\section{Conclusion}

Adult-onset autoimmune diabetes is prevalent and likely far more prevalent than childhood-onset Type 1 diabetes. Most cases of adultonset autoimmune diabetes are non-insulin requiring, i.e. LADA. In this review, we tried to highlight the genetic and immunological factors that play a major role in the predisposition of adult onset autoimmune diabetes. LADA and childhood-onset Type 1 diabetes are scarcely recognizable immunologically, in spite of the fact that the latter has a more noteworthy immunogenetic function with increasingly different DAA, more frequent IA- $2 \mathrm{~A}$ and $\mathrm{ZnT} 8 \mathrm{~A}$, in addition to lower $\mathrm{C}$ - peptide and increasingly quick $\mathrm{C}$-peptide loss. LADA is associated with several genetic markers, such as- PTPN22, STAT4, CTLA4, IL2RA, HLA, INS, SH2B3, ERBB3 and CLEC16A. LADA sufferers have distinct metabolic features with lower residual $\beta$-cell function than type 2 diabetic sufferers. The presence of any one of the autoantibodies in LADA patients as cited above leads to pancreatic $\beta$-cell failure besides T-cells which are largely responsible for inflammatory $\beta$ cell destruction among which GADA is the most dominant autoantibody. LADA sufferers have BMI in the overweight or obese categories $\left(\mathrm{BMI}>25.0 \mathrm{~kg} / \mathrm{m}^{2}\right)$. The best way to confirm LADA is via GAD antibody test and $\mathrm{HbA1c}$ test. Besides insulin treatment, the three DPP-4 inhibitors (sitagliptin, linagliptin and saxagliptin) can retain residual beta-cell function. Eventually, we can conclude from the aforesaid figures and facts that we have some anthropogenic characteristics that can be beneficial for the preliminary screening of LADA sufferers in a diabetic population, confirmatory diagnostic markers for LADA take C-peptide levels and GAD autoantibodies into consideration.

\section{Acknowledgments}

None.

\section{Conflicts of interest}

The authors declare that there is no conflict of interest.

\section{References}

1. American Diabetes Association. Diagnosis and classification of diabetes mellitus. Diabetes Care. 2010n;33(Suppl 1):S62-S69.

2. Romesh Khardori. Type 1 Diabetes Mellitus. Medscape. 2018.
3. Bluestone JA, Herold K, Eisenbarth G. Genetics, pathogenesis and clinical interventions in type 1 diabetes. Nature. 2010;464(7293):1293-1300.

4. Diagnosis of Diabetes and Prediabetes. National Institute of Diabetes and Digestive and Kidney Diseases. 2016.

5. Risérus U, Willett WC, Hu FB. Dietary fats and prevention of type 2 diabetes. Progress in Lipid Research. 2009;48(1):44-51.

6. Laugesen E, Ostergaard JA, Leslie RDG. Latent autoimmune diabetes of the adult: current knowledge and uncertainty. Diabet Med. 2015;32(7):843852 .

7. Stenström G, Gottsäter A, Bakhtadze E, et al. Latent autoimmune diabetes in adults: definition, prevalence, beta-cell function, and treatment. Diabetes. 2005;54(2):S68-72.

8. Pipi E, Marketou M, Tsirogianni A. Distinct clinical and laboratory characteristics of latent autoimmune diabetes in adults in relation to type 1 and type 2 diabetes mellitus. World J Diabetes. 2014;5(4):505-10.

9. David Spero BSN RN. LADA Diabetes Symptoms and Treatment. Diabetes self management. 2017.

10. Paolo Pozzilli, Silvia Pieralice. Latent Autoimmune Diabetes in Adults: Current Status and New Horizons. Endocrinol Metab. 2018;33(2):147159.

11. Joanna MM, Howson, Silke Rosinger, et al. Genetic Analysis of AdultOnset Autoimmune Diabetes. American Diabetes Association. Diabetes. 2011;60(10):2645-2653.

12. Dultz G, Matheis N, Dittmar M, et al. The protein tyrosine phosphatase non-receptor type $22 \mathrm{C} 1858 \mathrm{~T}$.polymorphism is a joint susceptibility locus for immune thyroiditis and autoimmune diabetes. Thyroid. 2009;19:143148.

13. Garth L Burn, Lena Svensson, Cristina Sanchez-Blanco, et al. Why is PTPN22 a good candidate susceptibility gene for autoimmune disease? FEBS Lett. 2011;585(23):3689-3698.

14. Korman BD, Kastner DL, Gregersen PK, et al. STAT4: genetics, mechanisms, and implications for autoimmunity. Curr Allergy Asthma Rep. 2008;8(5):398-403.

15. Amalia Lamana, Mercedes López-Santalla, Raquel Castillo-González, et al. The Minor Allele of rs7574865 in the STAT4 Gene Is Associated with Increased mRNA and Protein Expression. Plos One. 2015

16. Zeynep Yesim Kucuk, Louis-Marie Charbonnier, Jack JH. Bleesing, CTLA-4 Haplo-insufficiency in a Patient with an Autoimmune Lymphoproliferative Disorder. J Allergy Clin Immunol. 2017;140(3):862-864.e4.

17. Andersen MK, Lundgren V, Turunen JA, et al. Latent autoimmune diabetes in adults differs genetically from classical type 1 diabetes diagnosed after the age of 35 years. Diabetes Care. 2010;33:2062-2064.

18. Leonard WJ, Donlon TA, Lebo RV, Greene WC. Localization of the gene encoding the human interleukin-2 receptor on chromosome 10. Science. 1985;228(4707):1547-9.

19. Entrez Gene: INS insulin. Gene ID: 3630. 2018

20. Cerrone GE , Caputo M, Lopez AP , González C , et al . Variable number of tandem repeats of the insulin gene determines susceptibility to latent autoimmune diabetes in adults. Pub med. 2008;8(1):43-49.

21. Bimota Nambam, Shakti Aggarwal, Anju Jain. Latent autoimmune diabetes in adults: A distinct but heterogeneous clinical entity. World J Diabetes. 2010;1(4):111-115. 
22. Cervin C, Lyssenko V, Bakhtadze E, et al. Genetic similarities between latent autoimmune diabetes in adults, type 1 diabetes, and type 2 diabetes. Diabetes. 2008;57(5):1433-1437.

23. Bottazzo GF, Florin-Christensen A, Doniach D. Islet-cell antibodies in diabetes mellitus with autoimmune polyendocrine deficiencies. Lancet. 1974;2(7892):1279-83.

24. Hillman M, Torn C, Thorgeirsson H, et al. IgG4-subclass of glutamic acid decarboxylase antibody is more frequent in latent autoimmune diabetes in adults than in type 1 diabetes. Diabetologia. 2004;47(11):1984-9.

25. Turner R, Stratton I, Horton V, et al. UKPDS 25: autoantibodies to isletcell cytoplasm and glutamic acid decarboxylase for prediction of insulin requirement in type 2 diabetes. UK Prospective Diabetes Study Group. Lancet. 1997;350:1288-1293.

26. Vito Lampasona, Antonio Petrone, Claudio Tiberti, et al. Zinc Transporter 8 Antibodies Complement GAD and IA-2 Antibodies in the Identification and Characterization of Adult-Onset Autoimmune Diabetes. Non Insulin Requiring Autoimmune Diabetes (NIRAD). Diabetes Care. 2010;33(1):104-108.

27. Li Y, Zhao M, Hou C, et al. Abnormal DNA methylation in CD4 + T cells from people with latent autoimmune diabetes in adults. Diabetes Res Clin Pract. 2011;94(2):242-248.

28. Yang Z1, Zhou Z, Huang G, et al. The CD4(+) regulatory T-cells is decreased in adults with latent autoimmune diabetes. Diabetes Res Clin Pract. 2007;76(1):126-31

29. Padmos RC, Schloot NC, Beyan H, et al. Distinct monocyte geneexpression profiles in autoimmune diabetes. Diabetes. 2008;57(10):27682773

30. Yang L, Zhou Z, DU T, et al. Detection of carboxypeptidase H specific $\mathrm{T}$ cells in peripheral blood of latent autoimmune diabetic patients with carboxypeptidase antibody positivity by ELISPOT assay. Zhong Nan Da Xue Xue Bao Yi Xue Ban. 2009;34:1011-1016.

31. Roma Lightsey, RN, BSN. Diagnosis and treatment of latent autoimmune diabetes in adults still evolving. Clinical adv. 2011

32. Landin-Olsson M, Karlsson FA, Lernmark A, et al. The Diabetes Incidence Study in Sweden Group: Islet cell and thyrogastric antibodies in 633 consecutive 15-34 yr-old patients in the Diabetes Incidence Study in Sweden. Diabetes. 1992;41:1022-1027.

33. Borg H, Arnqvist HJ, Bjork E, et al. Evaluation of the new ADA and WHO criteria for classification of diabetes mellitus in young adults people (1534 yrs) in the Diabetes Incidence Study in Sweden (DISS). Diabetologia. 2003;46(2):173-181

34. Gottsäter A, Landin-Olsson M, Fernlund P, et al. Beta-cell function in relation to islet cell antibodies during the first $3 \mathrm{yr}$ after clinical diagnosis of diabetes in type II diabetic patients. Diabetes Care. 1993;16(6):902-910.
35. Fourlanos S, Dotta F, Greenbaum CJ, et al. Latent autoimmune diabetes in adults (LADA) should be less latent. Diabetologia. 2005;48(11):22062212 .

36. PozzilliP,DiMarioU.Autoimmunediabetesnotrequiring insulinat diagnosis (latent autoimmune diabetes of the adult): definition, characterization, and potential prevention. Diabetes Care. 2001;24(8):1460-1467.

37. Leslie RD, Pozzilli P. Type I diabetes masquerading as type II diabetes. Possible implications for prevention and treatment. Diabetes Care. 1994; 17:1214-1219.

38. Zinman B, Kahn SE, Haffner SM, et al. Phenotypic characteristics of GAD antibody-positive recently diagnosed patients with type 2 diabetes in North America and Europe. Diabetes. 2004;53(12):3193-3200.

39. Lohmann T, Nietzschmann U, Kiess W. Lady-like: is there a latent autoimmune diabetes in the young? Diabetes Care. 2000;23:1707-1708.

40. Thanh Nguyen, Tara L. Muzyk. LADA: A Little Known Type of Diabetes. Pharm time. 2009.

41. Gunnar Stenström, Anders Gottsäter, Ekaterine Bakhtadze, et al. Latent Autoimmune Diabetes in Adults-Definition, Prevalence, $\beta$-Cell Function, and Treatment. Diabetes. 2005;54(suppl 2): S68-S72.

42. Hagopian WA, Karlsen AE, Gottsater A, et al. Quantitative assay using recombinant human islet glutamic acid decarboxylase (GAD65) shows that 64K autoantibody positivity at onset predicts diabetes type. J Clin Invest. 1993;91(1):368-374.

43. Paolo Pozzilli, Silvia Pieralice. Latent Autoimmune Diabetes in Adults: Current Status and New Horizons. Endocrinol Metab (Seoul). 2018;33(2):147-159.

44. Isomaa B, Almgren P, Henricsson M, et al. Chronic complications in patients with slowly progressing autoimmune type 1 diabetes (LADA). Diabetes Care. 1999;22:1347-1353.

45. Myhill P, Davis WA, Bruce DG, et al. Chronic complications and mortality in community-based patients with latent autoimmune diabetes in adults: the Fremantle Diabetes Study. Diabet Med. 2008;25:1245-1250.

46. Lu J, Hou X, Zhang L, et al. Associations between clinical characteristics and chronic complications in latent autoimmune diabetes in adults and type 2 diabetes. Diabetes Metab Res Rev. 2015;31:411-420.

47. Isomaa B, Almgren P, Henricsson M, et al. Chronic complications in patients with slowly progressing autoimmune type 1 diabetes (LADA). Diabetes Care. 1999;22:1347-1353. 